\title{
Assessment of Anesthesiologists' Rapid Sequence Induction Technique in an University Hospital
}

\author{
Ursula Bueno do Prado Guirro, TEA ${ }^{1}$, Cesar Romão Martins, TSA ${ }^{2}$, Masashi Munechika, TSA 3
}

\begin{abstract}
Summary: Guirro UBP, Martins CR, Munechika M - Assessment of Anesthesiologists' Rapid Sequence Induction Technique in a University Hospital.

Background and objectives: The induction of the general anesthesia in patients on "a full stomach" can result in regurgitation of the gastric content and pulmonary aspiration. The function of the rapid sequence induction (RSI) is to minimize the time interval between the loss of the airway protection reflexes and tracheal intubation tube balloon. The objective of this study was to evaluate the rapid sequence induction among the anesthesiologists of the São Paulo Hospital.
\end{abstract}

\begin{abstract}
Methods: The participants answered the questionnaire voluntarily and anonymously, after signed consent. The questionnaire consisted of 60 questions about the fundamental points of the RSI. The questions were divided in pre-oxygenation, circuits, drugs used in the induction (opioids, hypnotics, neuromuscular blockers), cricoid pressure techniques, intubation and difficult intubation.
\end{abstract}

Results: Seventy-five questionnaires were applied and 22 were discarded due to incomplete answering. All anesthesiologists always declare doing pre-oxygenation and administering opioid, hypnotic, and neuromuscular blocker. Most use fentanyl (83\%), propofol (74.5\%) and succinylcoline $(68.6 \%)$. All anesthesiologists apply cricoid pressure. Most did not know the correct pressure to be applied on the cricoid cartilage. Intubation failures have already occurred with $71.7 \%$ of anesthesiologists and with $40 \%$, the regurgitation. When faced with an unexpected difficult intubation, anesthesiologists ask for the laryngeal mask (35.5\%).

Conclusions: This study showed a broad individual variety of the RSI technique, a fact already reported by different authors. The difficulty in establishing a RSI protocol can be attributed to constant evidence that science provides us, where updating over the years becomes good medical practice.

Keywords: Airway Management; Emergencies; Intubation, Intratracheal; Laryngopharyngeal Reflux; Respiratory Aspiration.

\section{INTRODUCTION}

The induction of the general anesthesia in patients on "a full stomach" or with an incompetent superior esophagus sphincter can result in regurgitation of the gastric content and pulmonary aspiration. The objective of rapid sequence induction (RSI) is to minimize the time interval between the loss of airway protective reflexes and balloon tube tracheal intubation ${ }^{1-3}$.

In 1946, Mendelson ${ }^{4}$ related food intake and gastric content reflux with the loss of airway protective reflexes and pulmonary aspiration to general anesthesia. The consequence of

Received from the São Paulo Hospital - Universidade Federal de São Paulo (UNIFESP), Brazil.

1. Master's, Clinical Surgery, Universidade Federal do Paraná (UFPR); Anesthesiologist, Worker's Hospital

2. Anesthesiologist, SAMMEDI; Medical Residency Preceptor, Teaching and Training Center of the Public Server's State Hospital of São Paulo

3. Assistant Professor, Anesthesiology, Pain and Intensive Medicine Discipline, Universidade Federal de São Paulo (UNIFESP)

Submitted on April 19, 2011

Approved on August 3, 2011

Correspondence to:

Úrsula Guirro, MD

Rua Mal. J. B. Bormann, 1373, ap. 1602

Batel

80730350 - Curitiba, PR, Brazil

E-mail: ursulaguirro@gmail.com bronchoaspiration is chemical pneumonitis, a severe inflammatory reaction of the pulmonary parenchyma. The protection of the airway against pulmonary aspiration is considered safe anesthetic practice.

The description of the induction technique in rapid sequence (RSI), with the goal of protecting the airway of aspiration of the digestive content in patients running this risk, improved and evolved since the introduction of succinilcoline - a depolarizing neuromuscular blocker used clinically since $1951^{6-7}$ - and since the first description of the cricoid pressure maneuver done by Sellick in $1961^{8}$. The rapid paralyzation of the vocal cords and cardiovascular stability established rocuronium for use in RSI. However, the duration of the prolonged effect with an adequate dose $\left(1.2 \mathrm{mg} \mathrm{kg}^{-1}\right)$ would cause limitation in its use. With the introduction of the sugammadex in the clinical practice in Europe, in 2005, and in Brazil, in 2010, studies to evaluate this drug's role in RSI began ${ }^{9}$.

RSI is broadly used in urgency and emergency anesthetic procedures, in patients with incomplete fasting and in obstetrics ${ }^{1-4}$. It is also used in situations where, although there is no emergency, the risk of aspiration is high, as in diabetic patients with gastroparesis, gastroesophageal reflux disease and in digestive obstructive cases ${ }^{1-3,9}$.

The "traditional" RSI technique consists in the positioning of the patient, pre-oxygenation, rapid injection of the pre-determined dose of the hypnotic, followed by the rapid-action 
neuromuscular blocker, pressure on the cricoid cartilage, avoiding positive pressure ventilation (PPV) before the tracheal intubation with the balloon tube ${ }^{3,11,12}$. However, the "traditional" RSI underwent variation with time and the medical practice, which prevented the creation of a standard protocol for the RSI ${ }^{3}$.

\section{METHODS}

After authorization from the Research Ethics Committee of UNIFESPIEPM, we administered the questionnaire to the anesthesiologists of the São Paulo Hospital (HSP) between March and April, 2007. All of the participants answered voluntarily and anonymously, after signed consent.

The questionnaire consisted of 60 questions about the fundamental points of RSI. The questions were divided in: preoxygenation, circuits, drugs used in the induction (opioids, hypnotics, and neuromuscular blockers), cricoid pressure techniques, intubation and difficult intubation.

The questions were answered individually, based on the experience of the anesthesiologist. The estimated time for the answers was 10 minutes. The data were tabled and submitted to descriptive statistical analysis.

\section{RESULTS}

Seventy-five questionnaires were applied. From these, 53 were adequately answered by the anesthesiologists and 22 were discarded due to more than $50 \%$ of questions left unanswered.

Anesthesiologists reported, on average, 11.3 \pm 8.1 years of experience in their specialty.

All of the anesthesiologists always offer pre-oxygenation. The pre-oxygenation technique chosen was normal respiration (54\%). The circuit of choice for most anesthesiologists to complete pre-oxygenation was the circular one (82.3\%). Regarding the flux of fresh gases used in pre-oxygenation, anesthesiologists of the HSP preferred the option between 4 and $6 \mathrm{~L}^{-\mathrm{m}^{-1}}(44.2 \%)$. The fraction of the $100 \% \mathrm{O}_{2}\left(\mathrm{FiO}_{2}\right)$ administered during the pre-oxygenation was chosen by all of the anesthesiologists (Table I).

The anesthesiologists of the HSP position the patients for $\mathrm{RSI}$ in proclive (46.1\%) or horizontal dorsal position (44.2\%). Only $5.8 \%$ answered that they do not worry about the positioning of the patient, and $3.8 \%$, in Trendelemburg.

All of the anesthesiologists reported to always administer an opioid during RSI. Most use fentanyl (83\%), followed by alfentanil (9.4\%), sufentanil (5.7\%) and remifentanil $(1.9 \%)$.

All of the anesthesiologists reported always administering a hypnotic in RSI. Most of the anesthesiologists chose propofol $(74.5 \%)$, followed by etomidate $(21.6 \%)$ and midazolam (3.9\%). None of the anesthesiologists reported to chose thiopental or ketamine.

All of the anesthesiologists reported always administering neuromuscular blockers (NMB) for RSI. The succinylcholine was chosen, preferentially by $68.6 \%$, followed by rocuronium
Table I - Pre-oxygenation

\begin{tabular}{|c|c|c|}
\hline \multicolumn{3}{|c|}{ Pre-oxynation technique } \\
\hline Normal respiration & 27 & $54 \%$ \\
\hline Deep respiration & 12 & $24 \%$ \\
\hline $\mathrm{SatO}_{2}$ up to $99 \%$ & 7 & $14 \%$ \\
\hline Vital capacity & 4 & $8 \%$ \\
\hline Others & 5 & $10 \%$ \\
\hline Total & 50 & $100 \%$ \\
\hline \multicolumn{3}{|l|}{ Circuits } \\
\hline Circular & 42 & $82.3 \%$ \\
\hline KT-5 & 9 & $17.7 \%$ \\
\hline Ambu & - & - \\
\hline Mask with storage & . & $\cdot$ \\
\hline Total & 51 & $100 \%$ \\
\hline \multicolumn{3}{|l|}{ Fresh gases flux } \\
\hline 8 L.m. $\mathrm{m}^{-1}$ or more & 6 & $11.6 \%$ \\
\hline 6 a 8 L.m $\mathrm{m}^{-1}$ & 8 & $15.4 \%$ \\
\hline 4 a 6 L.m $\mathrm{m}^{-1}$ & 23 & $44.2 \%$ \\
\hline 2 a 4 L.m-1 & 14 & $26.9 \%$ \\
\hline 1 a 2 L. $\mathrm{m}^{-1}$ & 1 & $1.9 \%$ \\
\hline Total & 52 & $100 \%$ \\
\hline \multicolumn{3}{|c|}{ Fraction of inhaled oxygen $\left(\mathrm{FiO}_{2}\right)$} \\
\hline $100 \%$ & 48 & $100 \%$ \\
\hline $90 \%$ & - & - \\
\hline $80 \%$ & - & - \\
\hline $70 \%$ & - & - \\
\hline $60 \%$ & - & - \\
\hline$\leq 50 \%$ & . & . \\
\hline TOTAL & 48 & $100 \%$ \\
\hline
\end{tabular}

chosen by $31.4 \%$. When they were questioned about the reason they preferentially use a certain NMB, most $(80 \%)$ chose the "beginning of the action", followed by the "prediction difficulties in intubation" (12\%) and "side effects of other NMB" (8\%).

The anesthesiologists of the HSP were questioned on whether they always do the cricoid pressure, or Sellick maneuver, during the RSI. All of the anesthesiologists reported positively. They were also questioned about who they asked for help when carrying out the cricoid pressure during the RSI. Most of the time, the help came from another doctor $(75.5 \%)$, followed by the nurses $(24.6 \%)$.

As for the moment in which they started the cricoid pressure in the RSI, the options were: on the awake, sleepy or unconscious patient. Forty nine per cent of the anesthesiologists began the cricoid pressure on the unconscious patient, $47.2 \%$ when the patient was sleepy and $3.8 \%$ when the patient was awake. When they were asked whether they knew the correct pressure to be applied on the cricoid, only $23.1 \%$ of the anesthesiologists answered that they knew, against $76.9 \%$ that reported they did not. Of the ones who said they knew the correct pressure, only two were correct (3.8\%).

When questioned about when to release the cricoid pressure, most of the anesthesiologists (69.2\%) released when the intubation was confirmed and the balloon was inflated, which 
Table II - When should the cricoid pressure be released in the RSI?

\begin{tabular}{lll}
\hline $\begin{array}{l}\text { Cricoid pressure: when should the cricoid pressure be } \\
\text { released in the RSI? }\end{array}$ & \multicolumn{3}{l}{} \\
\hline Confirmed intubation and inflated balloon & 36 & $69.2 \%$ \\
Inflated balloon & 16 & $30.8 \%$ \\
When the tube enters the trachea & - & - \\
Total & 52 & $100 \%$ \\
\hline
\end{tabular}

is considered correct. However, an alarming $30.8 \%$ of the anesthesiologists released the cricoid pressure on an inadequate moment: when only the balloon is inflated (Table II).

The anesthesiologists from the HSP were questioned about several aspects of the tracheal intubation technique and its complications during RSI. About intubation failure, most of the anesthesiologists (71.7\%) already experienced this urgency some time during a RSI. From the ones who mentioned a previous failure, the answer "only one" occurred more frequently $(47.1 \%)$, followed by "three or more" $(32.3 \%)$, and "two" (20.6\%). The anesthesiologists also answered about regurgitation episodes during RSI, indicating whether they had already witnessed it, how many times and what conduct they chose to carry out. Of the anesthesiologists, $40 \%$ stated they already had regurgitation. From these, $75 \%$ reported only one episode, and $25 \%$, two episodes or more. On the occasion, $58.6 \%$ aspirated their patients, $21.9 \%$ answered "other", and $19.5 \%$ positioned their patients in lateral position.

Anesthesiologists from the HSP were questioned about which device they ask for when faced with an unexpected and difficult intubation case during RSI. Their responses were they use a laryngeal mask in $35.5 \%$, followed by a new attempt with a guide $(20 \%)$, bronchoscopy $(15.5 \%)$, airway surgery $(6.7 \%)$, retrograde intubation $(6.7 \%)$, Sellick ventilation $(6.7 \%)$, bougie $(4.4 \%)$ and others $(4.4 \%)$.

\section{DISCUSSION}

This study has shown a broad individual variety of the RSI technique among anesthesiologists from the São Paulo Hospital, fact already reported by other authors. Morris et al. ${ }^{1}$ applied a questionnaire to the anesthesiologists and resident doctors of the same specialty about the characteristics of RSI and its complications, identifying great variation on the technique. More recently, Koerber ${ }^{12}$ also identified variations persistent on the ISR technique. The broad differences in ISR techniques practiced are possibly due to the absence of standard protocols in the institutions and medical literature.

From the studied sample in the HSP, all of the anesthesiologists always did pre-oxygenation, with various techniques. The pre-oxygenation with $100 \%$ oxygen fraction should always be carried out in order to guarantee patient safety, and avoid hypoxemia and desaturation, which generate an unnecessary risk of ventilating a patient without protect airways. The different existing oxygenation techniques have the goal of producing denitrogenation prior to the apnea period during the induction, displacing the alveolar nitrogen and substitution for oxygen. Thus, it allows the increase in the oxygen reserve and slows the beginning of the hypoxemia and arterial desaturation. The adequate time for pre-oxygenation varies between 2 to 7 minutes, for any technique that avoids the re-inhalation. The more modern equipments of anesthesia, coupled well with the mask, allow for an evaluation of the oxygen and nitrogen exhaled. The lack of adequate facial mask coupling cannot be compensated for the increase in time of oxygenation. Although all anesthesiologists knew that they should attach the mask well to the patient's face, in order to not allow the entrance of environment air in the fresh gas mixture, not all of them would do it rigorously $1,3,4,13$.

Most anesthesiologists of the HSP position the patients in proclive or horizontal dorsal for the RSI. The scientific literature is very divergent about the positioning. Moro et al. ${ }^{2}$ affirmed that the patients should be placed in proclive, with the dorsum elevated in 30 degrees during the induction, in order to avoid vomiting. If vomiting occurs, the position should be immediately altered to head down, to avoid bronchoaspiration. Those who are against this practice affirm that the vomit is active, and the gastric material could reach the larynx and aspiration would be inevitable due to gravity. Others, still, affirm that the head down position would be advantageous, because the gastric material would not reach the trachea and it would be expelled naturally. There are a few that affirm that the neutral position would be easier to intubate $2,3,14$.

Opioids are almost always administered as a part of the RSI; however, they are not part of the traditional technique ${ }^{10}$. The older opioids had a slow beginning of action and were associated with haemodynamic instability, different from the new opioids. A reduction of the sympathetic stimulation to the laryngoscopy is attributed to the opioids; a combination with other supporting drugs, such as hypnotics, betablockers or lidocaine, seems to be more effective. For adequate cardiovascular protection, opioids should be administered minutes before the induction of the anesthesia. This can promote ventilatory depression and ineffective denitrogenation, being inappropriate in urgency and emergency scenarios. The administration of fentanyl $\left(2 \mu \mathrm{g} \cdot \mathrm{kg}^{-1}\right)$, before the thiopental, propofol or etomidate, and succinycholine resulted in a more stable RSI than those without fentanyl. The alfentanil and the remifentanil have a faster beginning of action and seem to diminish the response to the laryngoscopy and intubation. In healthy patients, alfentanil $\left(30 \mu \mathrm{g} \cdot \mathrm{kg}^{-1}\right)$ almost completely weakened their response to the manipulation of the airways ${ }^{1-3,15-17}$. Possibly, the choice of fentanyl among anesthesiologists of the HSP is due to the medication being broadly available in the Brazilian market, having a low cost and being relatively well known by the doctors.

The ideal hypnotic agent should provide loss of consciousness effectively and rapidly, provide quality of intubation, hemodynamic stability, and diminish the sympathetic response to the laryngoscopy. Sadly, this drug still does not exist. The thiopental was exhaustively studied. Historically, it was the hypnotic agent of choice in a fixated dose; however, associ- 
ated with hemodynamic stability, thus the ketamine seem to be the induction agent of choice for patients with that condition. Nonetheless, there is still uncertainty about its safety in patients with intracranial hypertension. The midazolam has a slow beginning of action. The etomidate is the induction agent most used in emergencies, possibly due to the hemodynamic stability associated with its administration; thus, adrenocortical suppression is reported, even after only one dose, more pronounced and unwanted in septic patients. The propofol showed adequate conditions of intubation, weakening the laryngeal reflexes in a more efficient way than any other drug; however, with the disadvantage of hemodynamic instability. There are reports that the intubation conditions are not modified by the induction agent when associated with the administration of low doses of succinylcholine ${ }^{3,10,12,18-23}$. The preferred choice of HSP anesthesiologists was propofol, which is adequate in $\mathrm{RSI}$ and frequent among anesthesiologists.

The use of neuromuscular blockers (NMB) is a part of RSI. The succinylcholine was introduced in the clinical practice in 1951 and, despite its innumerous side effects, it continues to be used even today ${ }^{6,7}$. Its popularity is due to its fast beginning of action - between 30 and 60 seconds - and limited duration. The short-lasting action is due to the rapid hydrolysis by the butyrylcholinesterase enzyme. However, there are reports of deficiency of the enzyme and prolonged action ${ }^{24}$. The incidence of serious complications, such as malign hyperthermia, fatal hypercalcemia, bradyarrhythmias and the increase of intragastric and intraocular pressure, has led to a search for nondepolarizing -type agents without these unwanted effects, but with a similar latency and duration. The rocuronium, an aminosteroid type of neuromuscular blocker of intermediary duration, has been used in patients with "a full stomach" due to its fast set-up of blockage in the vocal cords and relative cardiovascular stability. The choice of almost $70 \%$ of anesthesiologists of the HSP was succinylcholine, motivated by one factor unaffected by pharmacodynamic: the beginning of action. When one wishes a short latency and tracheal intubation conditions similar to those found with the administration of succinylcholine, rocuronium in doses higher than $1.2 \mathrm{mg} \cdot \mathrm{kg}^{-1}$ should be used. Thus, these doses mean a longer action time, limiting its use in short procedures and mean a higher risk to patients with a possible difficult intubation, in the event that tracheal intubation fails. Since the introduction of sugammadex in the clinical practice in 2005 , the role of this drug in RSI has been studied ${ }^{1-3,9,25}$. Alternative techniques have been used with the goal of decreasing the latency period of the nondepolarizing NMB, such as the administration of NMB in divided doses, using a small dose of neuromuscular blocker a few minutes before its paralyzing dose or of another neuromuscular blocker. This technique was named priming and the subparalyzing dose, priming-dose. Although ineffective to produce neuromuscular blockage, this first dose would occupy a considerable number of postsynaptic receptors, allowing a faster appearance of the effect of the second dose of the neuromuscular blocker. Thus, this technique is not risk-free, having among its side effects: muscular weakness, difficulty swallowing, hypoventilation, reduction in coughing capacity, reduction of pulmonary volume, and the potential of deterioration in oxygenation. Relaxation of the muscles of the pharynx can occur, increasing the risk of gastric regurgitation. Until this moment, there is no evidence of the benefits of the use of the priming-dose ${ }^{1-3,26}$. The tracheal intubation without neuromuscular blockers should be reserved for those cases in which there are contraindication to the use of succinylcholine or use of nondepolaryzing neuromuscular blockers. It is important to highlight that inadequate conditions of intubation predispose trauma of the airway, intubation failure and difficulty of ventilation $1,2,27$.

The cricoids pressure has been studied exhaustively by many authors. Since Sellick's studies, when no patient vomited during the maneuver, the cricoid pressure was incorporated to the clinical practice. Thus, the authors questioned the maneuver, after reports of fatal aspirations occurred even when the technique was applied. It is impossible to determine to what extent these failures are related to an error in applying the maneuver. Some failures are due to the incorrect technique and uncertainty on when to start applying pressure, the adequate force and the moment to release. All of the anesthesiologists who participated in the research regularly perform the Sellick maneuver, but almost half of them begin the maneuver only after the loss of consciousness, which is not adequate, and practically no one knew the correct pressure to be applied in each moment. Evidence found in the literature is that cricoid pressure should begin when the patient is sleepy. Sellick ${ }^{8}$ described it as a "strong" pressure what is currently interpreted as $10 \mathrm{~N}(1 \mathrm{~kg})$ on a sleepy patient and $30 \mathrm{~N}(3 \mathrm{~kg})$ when loss of consciousness occurs. If a lesser pressure is applied, a pharyngeal occlusion may not occur, and, if the pressure is higher, there may be difficulty in intubation. There are no descriptions as to the use of right or left hand and it is recommended to ask for help of a trained assistant for the maneuver. The authors against the application of cricoid pressure affirm that an increase of aspiration risk would occur, and that the premature application would propitiate vomiting. Thus, the low morbidity of the Sellick maneuver is, possibly, greater than the potential risks of bronchoaspiration $3,8,28,29$. The maneuver should only be released when the intubation is confirmed and the balloon is inflated, or it results in risk of bronchoaspiration if the intubation is not correct.

The orotracheal intubation is a critical moment of RSI. Sadly, the difficulty with airway and intubation failure are more frequent during emergencies than in elective situations. Also, the clinician, when compared to anesthesiologists, seems to find greater difficulty in emergency intubations. This is an important discussion: would anesthesiologists have a greater intubating ability or create better conditions to carry it out? This discussion has been raised by Morris et al. ${ }^{1}$, in demonstrating that half of the interviewed anesthesiologists experienced at least one intubation failure in their career during a RSI, and at least a fourth of them were present when a regurgitation episode occurred. It is important to remember that the preoperatory evaluation in order to identify the airway is obligatory $1-3,30,31$. Of the evaluated anesthesiologists, around $70 \%$ had been present during an intubation failure and around $40 \%$ 
had been present during vomiting in the ISR. Facing intubation difficulties, anesthesiologists of the HSP answered that they first use the laryngeal mask, followed by a new attempt with a guide. None of the interviewed anesthesiologists answered that they would do an intubation in a patient awake or ventilation under the Sellick maneuver. Historically, the tendency is to avoid ventilating with positive pressure (PPV) during RSI, due to an alleged gastric insufflation and increase in risk of vomiting. More recently, some authors defended that ventilation under a mask and cricoid pressure limited to $20 \mathrm{~cm} \mathrm{H}_{2} \mathrm{O}$ would be acceptable in RSI. These authors believe that gastric insufflation would only occur when using the Sellick maneuver, with pressure reaching $45 \mathrm{~cm} \mathrm{H}_{2} \mathrm{O}$. Some authors report that, even without the cricoids pressure, the limited ventilation of $15 \mathrm{~cm} \mathrm{H}_{2} \mathrm{O}$ would be acceptable, because it would not distend the stomach. In fact, in Sellick's original article, he affirmed that the PPV and the cricoid pressure could be carried out without risk ${ }^{1-3,8,32}$. The risk of ventilating a patient with "a full stomach" does not justify the ordinary use of PPV, unless when associated with a potential benefit, as in intubation failure cases and progression for desaturation and hypoxemia, in which PPV is applied under the mask in association with cricoid pressure $3,33,34$. Low adhesion to the use of bougie in the HSP group should be highlighted, which presents low cost, easiness of use, and is a valuable alternative in unexpectedly difficult airway cases ${ }^{35}$.

\section{CONCLUSION}

The rapid sequence induction is indicated for those patients who are in risk of gastric aspiration. Previous evaluation of the patient, preparation of anesthetic material, aspirator, com- plete checking of the equipment, positioning of the patient, pre-oxygenation, drugs chosen and the correct application of the cricoid pressure are part of the technique and provide safety.

The difficulty in establishing a protocol for RSI can be attributed to the constant evidences that science gives us, making updating information over the years good medical practice.

The available drugs can foster excellent conditions for tracheal intubation with safety, promoting a short period of latency, fast return of consciousness and spontaneous ventilation, if necessary. The choice of drugs should be based on the patient's physical state, hemodynamic stability and the potential side effects. A PPV is only recommended in emergency cases, when there is intubation failure and clinical deterioration. The cricoid pressure, although controversial, is still recommended due to lack of evidence for not applying it.

The results found in this study point to the partial adhesion of anesthesiologists of the HSP to the recommended rapid sequence induction technique, with certain points to be improved according to the evolution of scientific evidences.

\section{ACKNOWLEDGMENTS}

We thank the anesthesiologists from the São Paulo Hospital who answered the questionnaire, voluntarily participating in this research and making a little contribution to our infinite knowledge in the art of Anesthesiology. Still, I thank Professor Dr. Maria Angela Tardelli for her countless contributions in the revision of questionnaires and to Professor Dr. Flavia Ribeiro Machado for reapplying the questionnaire in the Intensive Medicine environment. 


\section{REFERÊNCIAS/REFERÊNCIAS}

1. Morris J, Cook TM - Rapid sequence induction: a national survey of practice. Anesthesia, 2001;56:1090-1115.

2. Moro ET, Módolo NSP - Indução anestésica com a técnica de sequência rápida. Rev Bras Anestesiol, 2004;54(4):595-606.

3. El-Orbany M, Connolly LA - Rapid Sequence Induction and Intubation: Current Controversy. Anesth Analg, 2010;110(5):1318-1325.

4. Mendelson $\mathrm{CL}$ - The aspiration of stomach contents into the lungs during obstetric anesthesia. Am J Obstet Gynecol, 1946;52:191-205.

5. Brownlee IA, Aseeri A, Ward C et al. - From gastric aspiration to airway inflammation. Monaldi Arch Chest Dis, 2010;73(2):54-63.

6. Dorkins HR - Suxamethonium: The development of a modern drug from 1906 to the present day. Med Hist, 1982;26(2):145-168.

7. Almeida MCS - Succinilcolina: 50 anos de soberania. Rev Bras Anestesiol, 2002;52(4):513-516.

8. Sellick BA - Crycoid pressure to control regurgitation of stomach contents during induction of anesthesia. Lancet, 1961;19(2):404-406.

9. Bisschops MM, Holleman C, Huitink JM - Can sugammadex save a patient in a simulated 'cannot intubate, cannot ventilate' situation? Anaesthesia, 2010;65:936-941.

10. Stept WJ, Safar P - Rapid induction-intubation for prevention of gastric-content aspiration. Anesth Analg, 1970;49(4):633-636.

11. Suresh MS, Munnur U, Wali A - The patient with a full stomach. Em: Hagberg CA - Benumof's airway management: principles and practice. $2^{\mathrm{a}}$ Ed, Philadelphia, Mosby, 2007; pp. 764-766.

12. Koerber JP, Roberts GE, Whitaker R et al. - Variation in rapid sequence induction techniques: current practice in Wales. Anaesthesia, 2009;64(1):54-59.

13. Gregori WM, Mathias LAST, Piccinini Fo L et al. - Impacto do acoplamento de máscara facial sobre a oxigenação. Rev Bras de Anestesiol, 2005;55(5):500-507.

14. Goldberg JS - What is the correct position of the neck for rapid sequence induction in the patient with a "full stomach"? Anesthesiology, 1987;66(4):588. 
15. Harris CE, Murray AM, Anderson JM et al. - Effects of thiopentone, etomidate and propofol on the haemodynamic response to tracheal intubation. Anaesthesia, 1988;43(suppl):32-36.

16. Miller DR, Martineau RJ, OBrien $\mathrm{H}$ et al. - Effects of alfentanil on the hemodynamic and catecholamine response to tracheal intubation. Anesth Analg, 1993;76(5):1040-1046.

17. Farman $\mathrm{J}-$ Acid aspiration syndrome. $\mathrm{Br} \mathrm{J}$ of Perioper Nurs, 2004;14:266-267, 269-270, 272-274.

18. Lewis CB - Endotracheal intubation under thiopentone; an analysis of 200 cases. Anaesthesia, 1948;3(3):113-115.

19. El-Orbany MI, Joseph NJ, Salem MR - Tracheal intubating conditions and apnoea time after small dose of succinylcholine are not modified by the choice of induction agent. Br J Anaesth, 2005;95(5):710-714.

20. Yeung $\mathrm{JK}_{\perp}$ Zed $\mathrm{PJ} \_$- A review of etomidate for rapid sequence intubation in the emergency department. CJEM, 2002;4(3):194-198.

21. SwansonER,FosnochtDE, JensenSC-Comparisonofetomidateandmidazolam for prehospital rapid-sequence intubation. Prehosp Emerg Care, 2004;8(3):273-279.

22. Hohl $\mathrm{CM}$, Kelly-Smith $\mathrm{CH}$, Yeung TC et al. - The effect of a bolus dose of etomidate on cortisol levels, mortality, and health services utilization: a systematic review. Ann Emerg Med, 2010;56(2):105-113.

23. Albert SG, Ariyan S, Rather A - The effect of etomidate on adrenal function in critical illness: a systematic review. Intensive Care Med, 2011;4 [no prelo].

24. Kaufman SE, Donnell RW, Aiken DC et al. - Prolonged neuromuscular paralysis following rapid-sequence intubation with succinylcholine (April). Ann Pharmacother, 2011;22 [no prelo].

25. Perry JJ, Lee JS, Sillberg VAH et al. - Rocuronium versus succinylcholine for rapid sequence induction intubation. Cochrane Database of Syst Rev, 2008;16(2):CD002788.

26. Schmidt J, Irouschek A, Muenster T et al. - A priming technique accelerates onset of neuromuscular blockade at the laryngeal adductor muscles. Can J Anaesth, 2005;52(1):50-54.

27. Hawthorne L, Wilson R, Lyons $G$ et al. - Failed intubation revisited: 17-years experience in a teaching maternity unit. $\mathrm{Br} \mathrm{J}$ Anaesth, 1996;76(5):680-684.

28. Vanner RG, Asai T - Safe use of cricoid pressure. Anaesthesia, 1999;54(1):1-3.

29. Priebe $\mathrm{HJ}$ - Cricoid pressure: an experts opinion. Minerva Anestesiol, 2009;75(12):710-714.

30. Yamanaka CS, Góis AFT, Vieira PCB et al. - Intubação orotraqueal: avaliação do conhecimento médico e das práticas clínicas adotadas em unidades de terapia intensiva. Rev Bras Ter Intensiva, 2010;22(2):103-111.

31. Martin LD, Mhyre JM, Shanks AM et al. $-3,423$ emergency tracheal intubations at a university hospital: airway outcomes and complications. Anesthesiology, 2011;114(1):42-48.

32. Clements $\mathrm{P}$, Washington SJ, McCluskey A - Should patients be manually ventilated during rapid sequence induction of anaesthesia? $\mathrm{Br} \mathrm{J}$ Hosp Med, 2009;70(7):424.

33. Brown JP, Werrett G - Bag-mask ventilation in rapid sequence induction. Anaesthesia, 2009;64(7):784-785.
34. Rangasami J, Ponnusamy K - Rapid sequence induction. Guidelines rapid sequence induction, non-pregnant adult patient, no predicted difficulty from Difficult Airway Society of the United Kingdom. Disponível em: http://www.das.uk.com/guidelines/rsi.html (acessado em: 29/03/2011).

35. Reis LA, Reis GFF, Oliveira MRM et al. - Bougie. Rev Bras Anestesiol, 2009;59(5):618-623. 